\title{
Many-body localization from dynamical gauge fields
}

\author{
Zhiyuan Yao $\odot,{ }^{1}$ Chang Liu $\odot,{ }^{1}$ Pengfei Zhang,,${ }^{1,2,3}$ and Hui Zhai $\odot^{1, *}$ \\ ${ }^{1}$ Institute for Advanced Study, Tsinghua University, Beijing 100084, China \\ ${ }^{2}$ Institute for Quantum Information and Matter, California Institute of Technology, Pasadena, California 91125, USA \\ ${ }^{3}$ Walter Burke Institute for Theoretical Physics, California Institute of Technology, Pasadena, California 91125, USA
}

(Received 15 April 2020; accepted 8 September 2020; published 18 September 2020)

\begin{abstract}
A recent experiment [C. Schweizer, F. Grusdt, M. Berngruber, L. Barbiero, E. Demler, N. Goldman, I. Bloch, and M. Aidelsburger, Nat. Phys. 15, 1168 (2019)] has realized a dynamical gauge system with a $\mathbb{Z}_{2}$ gauge symmetry in a double-well potential. In this work we propose a method to generalize this model from a single double well to a one-dimensional chain. We show that although there are no disordered potentials in the original model, the phenomenon of many-body localization can occur. The key ingredient is that different symmetry sectors with different local gauge charges play the role of different disorder configurations, which becomes clear after exactly mapping our model to a transverse Ising model in a random longitudinal field. We show that both the ergodic regime and the many-body localized regime exist in this model from four different metrics, which include level statistics, volume law versus area law of entanglement entropy of eigenstates, quench dynamics of entanglement entropy, and physical observables.
\end{abstract}

DOI: 10.1103/PhysRevB.102.104302

\section{INTRODUCTION}

In the past decade, one of the main research topics in cold-atom physics is simulating synthetic gauge fields [1-3]. Two commonly used methods are utilizing Raman transitions and shaking optical lattices periodically. It first started with simulating a constant Abelian gauge field [4,5], which can be gauged out and does not have observable effects. Physical effects of gauge fields can be produced either by introducing a spatial or temporal dependence $[6,7]$ or by generalizing gauge fields from Abelian ones to non-Abelian ones [8-10]. By introducing spatial or temporal dependence, this protocol realizes synthetic magnetic fields [6] or synthetic electric fields [7], which manifest themselves as vortices in superfluids [6] and the Hall effect [11]. By generalizing to the non-Abelian case, spin-orbit couplings can be induced [12-14], which give rise to rich physics such as the stripe superfluids for Bose condensates [15-19] and topological bands for Fermi gases [20]. Nevertheless, the primary focuses in these studies are the properties of the matter fields since the gauge fields are fixed by external classical sources, such as laser fields and magnetic fields, and do not receive back-action from the matter fields.

In the second wave of research along this line, the gauge fields acquire their own dynamics. For example, recently density-dependent gauge fields have been created using lattice shaking, either by tuning the shaking frequency resonant with the interaction strength [21] or by periodically driving both the optical lattice and the interactions at the same frequency [22]. As we know, the density of a many-body system is a dynamical variable that can fluctuate spatially and temporally.

\footnotetext{
*hzhai@tsinghua.edu.cn
}

Therefore, such density-dependent gauge fields are dynamical. Nevertheless, this does not mean they are "dynamical gauge fields" as we understand in high-energy physics, where local gauge symmetries are indispensable. In other words, to be eligible for dynamical gauge fields, not only the gauge fields need to have their own dynamics, but the dynamical terms also have to be invariant under local gauge transformations. One familiar example is the Maxwell theory in which the dynamical term $-\frac{1}{4} \mathcal{F}_{\mu \nu} \mathcal{F}^{\mu \nu}$, where $\mathcal{F}^{\mu \nu}$ is the electromagnetic field tensor, is invariant under local $\mathrm{U}(1)$ gauge transformations.

Significant progresses have been made in recent experiments, and a zero-dimensional two-site $\mathbb{Z}_{2}$ version of dynamical gauge fields has been created [23]. This model possesses a $\mathbb{Z}_{2}$ gauge symmetry, although there is no distinction between "local" and "global" in the two-site case. In the experiment reported in Ref. [23], two ${ }^{87} \mathrm{Rb}$ atoms, one in hyperfine state $\left|F=1, m_{F}=1\right\rangle$ ( $f$ atom) and one in hyperfine state $\left|F=1, m_{F}=-1\right\rangle$ ( $a$ atom), are confined in a double-well potential. The creation and annihilation operators of $f$ atom $\left(a\right.$ atom) are denoted by $\hat{f}_{i}^{\dagger}\left(\hat{a}_{i}^{\dagger}\right)$ and $\hat{f}_{i}\left(\hat{a}_{i}\right)$, with $i=1,2$ for the two sites. Since the total number of $f$ atoms in the double well is conserved and fixed to be one, we can introduce a spin- $\frac{1}{2}$ operator $\hat{\boldsymbol{\tau}}_{i}$ for this double well defined as follows $(\hbar=1)$ :

$$
\begin{aligned}
& \hat{\tau}^{x}=\frac{1}{2}\left(\hat{f}_{1}^{\dagger} \hat{f}_{2}+\hat{f}_{2}^{\dagger} \hat{f}_{1}\right), \\
& \hat{\tau}^{y}=\frac{i}{2}\left(\hat{f}_{2}^{\dagger} \hat{f}_{1}-\hat{f}_{1}^{\dagger} \hat{f}_{2}\right), \\
& \hat{\tau}^{z}=\frac{1}{2}\left(\hat{f}_{1}^{\dagger} \hat{f}_{1}-\hat{f}_{2}^{\dagger} \hat{f}_{2}\right) .
\end{aligned}
$$

By shaking the optical lattice at some fine-tuned frequency, one can reach the following effective Hamiltonian to describe 
the coupling between the $a$ atom and the $f$ atom [23,24]:

$$
\hat{H}_{\mathrm{dw}}=-2 g \hat{\tau}^{z}\left(\hat{a}_{1}^{\dagger} \hat{a}_{2}+\hat{a}_{2}^{\dagger} \hat{a}_{1}\right)-2 h \hat{\tau}^{x} .
$$

This model exhibits a $\mathbb{Z}_{2}$ symmetry: the Hamiltonian is invariant under $\hat{\tau}^{z} \rightarrow-\hat{\tau}^{z}$ and simultaneously either $\hat{a}_{1} \rightarrow-\hat{a}_{1}$ or $\hat{a}_{2} \rightarrow-\hat{a}_{2}$.

However, there is a difficulty in extending this model from zero spatial dimension to higher dimensions. If we allow $f$ atoms to hop outside the double wells and their numbers to change, the definition of the $\hat{\boldsymbol{\tau}}_{i}$ operator [Eqs. (1)(3)] becomes invalid and, consequently, the $\mathbb{Z}_{2}$ symmetries are lost. If we allow $a$ atoms to hop outside of the double wells, it will render the local $\mathbb{Z}_{2}$ symmetries into a global one.

At this stage, two important questions arise for studying "dynamical gauge fields" with cold atoms. The first one is how to realize dynamical gauge field models with local gauge symmetries, especially, how to extend the doublewell models realized in Ref. [23] to include spatial degrees of freedom. The second one is, provided that such models of dynamical gauge fields have been realized using cold atoms, what are the unique physical effects, aside from those have been discussed in the context of high-energy physics. Recently, various attempts have been made to address these two issues [23-46]. For example, Barbiero et al. have proposed a way to link double wells together to form a two-dimensional square lattice with local $\mathbb{Z}_{2}$ symmetries [24].

In this work we offer our answers to these two questions. First, we propose a scheme to extend the double-well model (4) to one dimensional. The central idea of our proposal is to utilize the interactions between particles to build up the spatial dimension in a way that the local gauge symmetries are preserved. Second, we show that the phenomenon of many-body localization (MBL) can emerge in our dynamical gauge field model. Although MBL is usually studied in interacting disordered models [52,53], we show that the phenomena can also occur in our model which is disorder free. The key point is that different conserved local gauge charges associated with their local gauge symmetries play the role of disordered potentials in interacting disordered models of MBL, due to the couplings between the gauge fields and the matter fields. Recently, many-body localization due to local gauge symmetries has also been discussed in several works [43-46]. The model studied in this work is different from theirs and we also apply different metrics to distinguish MBL from thermalization.

\section{MODEL}

\section{A. One-dimensional dynamical gauge fields}

Here we consider two spatially overlapped onedimensional chains, each of which consists of a series of double wells, as sketched in Fig. 1. In the first chain, for every even number $i$, a double well is formed by the $i$ th site and $(i+1)$ th site, and each double well contains exactly one $a$ atom and one $f$ atom. This means for each double well with

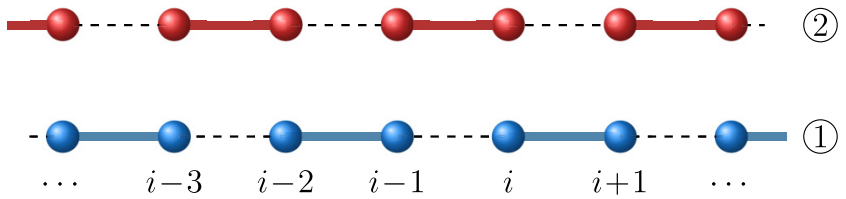

FIG. 1. Our model contains two chains with staggered double wells denoted by the solid blue and red bonds. The blue chain consists of $a$ and $f$ atoms, and the red chain is loaded with $b$ and $d$ atoms. Although for illustration purposes two chains are drawn separated in space, they, in reality, should overlap spatially to allow for interaction between atoms at the same site.

a pair of sites $i$ and $i+1$, we can introduce a spin- $\frac{1}{2}$ operator $\hat{\boldsymbol{\tau}}_{i}$ for the $f$ atom

$$
\begin{aligned}
& \hat{\tau}_{i}^{x}=\frac{1}{2}\left(\hat{f}_{i}^{\dagger} \hat{f}_{i+1}+\hat{f}_{i+1}^{\dagger} \hat{f_{i}}\right), \\
& \hat{\tau}_{i}^{y}=\frac{i}{2}\left(\hat{f}_{i+1}^{\dagger} \hat{f}_{i}-\hat{f}_{i}^{\dagger} \hat{f}_{i+1}\right), \\
& \hat{\tau}_{i}^{z}=\frac{1}{2}\left(\hat{f}_{i}^{\dagger} \hat{f}_{i}-\hat{f}_{i+1}^{\dagger} \hat{f}_{i+1}\right) .
\end{aligned}
$$

Similarly, we can introduce another spin- $\frac{1}{2}$ operator $\hat{\boldsymbol{\sigma}}_{i}$ for the $a$ atom:

$$
\begin{aligned}
& \hat{\sigma}_{i}^{x}=\frac{1}{2}\left(\hat{a}_{i}^{\dagger} \hat{a}_{i+1}+\hat{a}_{i+1}^{\dagger} \hat{a}_{i}\right), \\
& \hat{\sigma}_{i}^{y}=\frac{i}{2}\left(\hat{a}_{i+1}^{\dagger} \hat{a}_{i}-\hat{a}_{i}^{\dagger} \hat{a}_{i+1}\right), \\
& \hat{\sigma}_{i}^{z}=\frac{1}{2}\left(\hat{a}_{i}^{\dagger} \hat{a}_{i}-\hat{a}_{i+1}^{\dagger} \hat{a}_{i+1}\right),
\end{aligned}
$$

In this way, we have introduced $\hat{\boldsymbol{\tau}}_{i}$ and $\hat{\boldsymbol{\sigma}}_{i}$ for every even number $i$. The Hamiltonian of this chain is simply a sum of that of each individual double well, Eq. (4), and takes the form

$$
\hat{H}=\sum_{i=\text { even }}-4 g \hat{\tau}_{i}^{z} \hat{\sigma}_{i}^{x}-2 h \hat{\tau}_{i}^{x},
$$

in terms of previously defined spin- $\frac{1}{2}$ operators. This Hamiltonian has included the effect of lattice shaking, the hopping of both $a$ and $f$ atoms inside the double wells, and the interactions between $a$ and $f$ atoms $[23,24]$. It is exactly the same model as that has been realized in the recent experiment [23].

Next, we consider the second chain constituted with $b$ and $d$ atoms that play the same roles as $a$ and $f$ atoms in the first chain, respectively. Note that compared with $a$ and $f$ atoms, $b$ and $d$ atoms can be different atomic species and have different ac polarizabilities. Therefore, $b$ and $d$ atoms experience a different lattice potential from the other set of lasers such that, in the second chain, each double well is formed by the $i$ th and $(i+1)$ th sites with odd $i$. Similar to the first chain, these double wells are disconnected and each double well contains exactly one $b$ atom and one $d$ atom. We can, therefore, introduce two spin- $\frac{1}{2}$ operators $\hat{\boldsymbol{\tau}}_{i}$ and $\hat{\boldsymbol{\sigma}}_{i}$ for every odd number $i$. Their definitions are given by Eqs. (5)-(7) upon substituting $f$-atom operators with $d$-atom operators and by Eqs. (8)-(10) after replacing $a$-atom operators with $b$-atom operators. In this way, we have introduced $\hat{\boldsymbol{\tau}}_{i}$ and $\hat{\boldsymbol{\sigma}}_{i}$ for every odd number $i$. Moreover, the same shaking protocol is applied to this chain, which generates the same Hamiltonian as Eq. (11) except that the summation is over odd numbers of $i$. At this point, we have 
defined two sets of spin operators $\hat{\boldsymbol{\sigma}}_{i}$ and $\hat{\boldsymbol{\tau}}_{i}$ for all the links in this one-dimensional system.

Now, we turn on interactions between the two chains. We emphasize again that these two chains are arranged to be spatially overlapping with each other so that atoms at the same site can interact with each other. The interactions between $a$ and $f$ atoms and between $b$ and $d$ atoms have already been taken into account when writing the effective Hamiltonian (11). Since the interactions between atoms depend on the magnetic levels and the location of magnetic field, due to the presence of Feshbach resonances, one can choose a proper location of magnetic field such that the interaction between $a$ and $b$ atoms is much larger than others. Noting that for even number $i$, we have

$$
\begin{gathered}
\hat{a}_{i}^{\dagger} \hat{a}_{i}=1 / 2+\hat{\sigma}_{i}^{z} ; \\
\hat{b}_{i}^{\dagger} \hat{b}_{i}=1 / 2-\hat{\sigma}_{i-1}^{z} .
\end{gathered}
$$

And for odd number $i$, we have

$$
\begin{gathered}
\hat{a}_{i}^{\dagger} \hat{a}_{i}=1 / 2-\hat{\sigma}_{i-1}^{z} ; \\
\hat{b}_{i}^{\dagger} \hat{b}_{i}=1 / 2+\hat{\sigma}_{i}^{z} .
\end{gathered}
$$

The interactions between $a$ and $b$ atoms can then be cast into the form

$$
U \sum_{i} \hat{a}_{i}^{\dagger} \hat{a}_{i} \hat{b}_{i}^{\dagger} \hat{b}_{i}=-U \sum_{i}\left(\hat{\sigma}_{i}^{z} \hat{\sigma}_{i+1}^{z}-1 / 4\right)
$$

Hence, the total Hamiltonian of this system reads as

$$
\hat{H}=\sum_{i}\left(-4 g \hat{\tau}_{i}^{z} \hat{\sigma}_{i}^{x}-2 h \hat{\tau}_{i}^{x}-4 \hat{\sigma}_{i}^{z} \hat{\sigma}_{i+1}^{z}\right) .
$$

Here and hereafter, we set $U / 4=1$ as our energy unit, and $g$ and $h$ here are actually dimensionless numbers $4 g / U$ and $4 h / U$ in terms of the original parameters. Obviously, this Hamiltonian is invariant under the transformation $\hat{\sigma}_{i}^{x} \rightarrow-\hat{\sigma}_{i}^{x}$ and $\hat{\tau}_{i}^{z} \rightarrow-\hat{\tau}_{i}^{z}$, and possesses a local $\mathbb{Z}_{2}$ gauge symmetry. Our Hamiltonian can also be brought into the standard Ising gauge theory by the following duality transformation:

$$
\sigma_{i}^{z} \sigma_{i+1}^{z} \rightarrow \tilde{\sigma}_{i}^{x}, \quad \sigma_{i}^{x} \rightarrow \tilde{\sigma}_{i-1}^{z} \tilde{\sigma}_{i}^{z},
$$

where $\tilde{\sigma}_{i}$ is defined on the dual lattice. The dual Hamiltonian nows take the form

$$
\hat{H}=\sum_{i}\left(-4 g \hat{\tau}_{i}^{z} \hat{\tilde{\sigma}}_{i-1}^{z} \hat{\tilde{\sigma}}_{i}^{z}-2 h \hat{\tau}_{i}^{x}-4 \hat{\tilde{\sigma}}_{i}^{x}\right),
$$

which is the standard Ising gauge theory with $\mathbb{Z}_{2}$ matter fields [47].

\section{B. Mapping to a model of many-body localization}

For later convenience, we shall rotate $\hat{\boldsymbol{\tau}}_{i}$ as follows:

$$
\hat{\tau}_{i}^{x} \rightarrow \hat{\tau}_{i}^{z}, \quad \hat{\tau}_{i}^{y} \rightarrow-\hat{\tau}_{i}^{y}, \text { and } \hat{\tau}_{i}^{z} \rightarrow \hat{\tau}_{i}^{x} .
$$

The Hamiltonian then becomes

$$
\hat{H}=\sum_{i}\left(-4 g \hat{\tau}_{i}^{x} \hat{\sigma}_{i}^{x}-2 h \hat{\tau}_{i}^{z}-4 \hat{\sigma}_{i}^{z} \hat{\sigma}_{i+1}^{z}\right) .
$$

In this new basis, one can easily see that $\hat{Q}_{i}=4 \hat{\sigma}_{i}^{z} \hat{\tau}_{i}^{z}$ commutes with $\hat{H}$ and its eigenvalue $q_{i}$ takes the value of +1 or -1 . For any eigenstate |\rangle , we have $\hat{Q}_{i}|\rangle=q_{i}|\rangle$, and $q_{i}$ can be understood as the conserved local gauge charge associated with the local gauge symmetries. The four local bases of site $i$, $\left|\sigma_{i}^{z} \tau_{i}^{z}\right\rangle$, fall into two classes: $|\uparrow \uparrow\rangle$ and $|\downarrow \downarrow\rangle$ with $q_{i}=1$, and $|\uparrow \downarrow\rangle$ and $|\downarrow \uparrow\rangle$ with $q_{i}=-1$. To make use of the reduced local Hilbert space for given $q_{i}$, we introduce another spin- $\frac{1}{2}$ operator $\hat{\boldsymbol{\Gamma}}_{i}$ defined as

$$
\hat{\Gamma}_{i}^{x}=2 \hat{\tau}_{i}^{x} \hat{\sigma}_{i}^{x}, \quad \hat{\Gamma}_{i}^{y}=2 \hat{\tau}_{i}^{x} \hat{\sigma}_{i}^{y}, \quad \text { and } \quad \hat{\Gamma}_{i}^{z}=\hat{\sigma}_{i}^{z} .
$$

Indeed, these operators satisfy the $\mathrm{SU}(2)$ algebra: $\left[\hat{\Gamma}_{i}^{x}, \hat{\Gamma}_{i}^{y}\right]=$ $i \hat{\Gamma}_{i}^{z},\left[\hat{\Gamma}_{i}^{y}, \hat{\Gamma}_{i}^{z}\right]=i \hat{\Gamma}_{i}^{x}$, and $\left[\hat{\Gamma}_{i}^{z}, \hat{\Gamma}_{i}^{x}\right]=i \hat{\Gamma}_{i}^{y}($ note $\hbar=1)$. It can be verified that the spin-up and -down states, denoted as $|\Uparrow\rangle$ and $|\downarrow\rangle$, of the $\hat{\boldsymbol{\Gamma}}_{i}$ operator, are $|\uparrow \uparrow\rangle$ and $|\downarrow \downarrow\rangle$, respectively, for the subspace with $q_{i}=1$, and are $|\uparrow \downarrow\rangle$ and $|\downarrow \uparrow\rangle$, respectively, for the subspace with $q_{i}=-1$. Noticing that

$$
\hat{\tau}_{i}^{z}=4 \hat{\tau}_{i}^{z} \hat{\sigma}_{i}^{z} \hat{\sigma}_{i}^{z}=\hat{Q}_{i} \hat{\Gamma}_{i}^{z},
$$

we can always replace $\hat{\tau}_{i}$ by $q_{i} \hat{\Gamma}_{i}$ in the Hamiltonian when it acts on states in a symmetry sector with given $\left\{q_{i}\right\}$. With the help of the definition of the $\hat{\boldsymbol{\Gamma}}_{i}$ operators, the Hamiltonian in this symmetry sector can be simplified to

$$
\hat{H}=\sum_{i}\left(-2 g \hat{\Gamma}_{i}^{x}-2 h q_{i} \hat{\Gamma}_{i}^{z}-4 \hat{\Gamma}_{i}^{z} \hat{\Gamma}_{i+1}^{z}\right) .
$$

Because $q_{i}$ takes \pm 1 in different symmetry sectors, $h q_{i}$ can be either $+h$ or $-h$ and acts as a $\mathbb{Z}_{2}$ random field. In this way, we map our model to a transverse Ising model in the presence of a disordered longitudinal field. This mapping clearly reveals that the local conserved gauge charges play the role of disordered potentials. Since the gauge charge is always quantized, the difference between this model and usual models of MBL is the distribution of the random field. In a usual model of MBL, the field takes a continuous distribution, say, uniformly distributed in $[-h, h]$, whereas in our effective model, the field takes two discrete values with equal probabilities. Nevertheless, this difference does not exclude the possibility of MBL. In the weak disorder limit $h \ll g$, the system is ergodic and obeys the eigenstate thermalization hypothesis (ETH) [48-51]. In the opposite strong disorder limit $h \gg g$, ETH fails and the system is expected to be in the MBL regime [52,53]. Below we will demonstrate the presence of both the ETH (thermal) regime and the MBL regime in this model from several different metrics.

\section{MANY-BODY LOCALIZATION}

The differences between the ETH regime and the MBL regime can be characterized using a number of different physical quantities [51,54-56]. In this section, we will employ four different metrics, related to the properties of eigenstate and the behavior of quench dynamics from a product state listed in Fig. 2. 


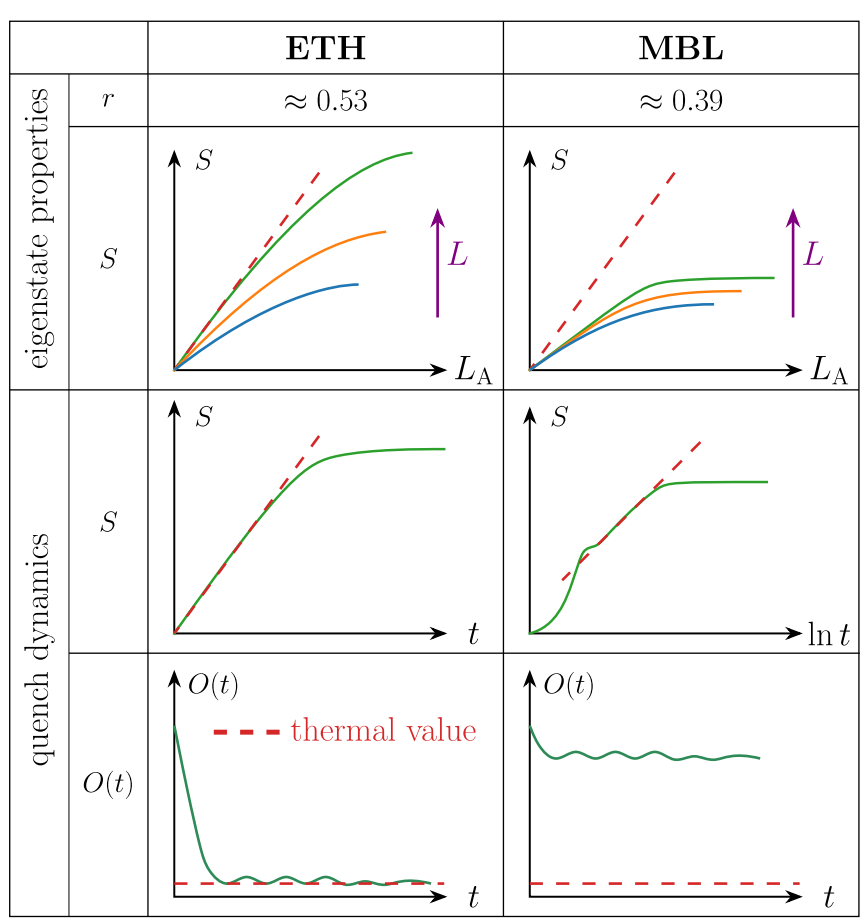

FIG. 2. Summary of four metrics to distinguish a thermal (ETH) phase from a MBL phase: (i) $r$, which is a quantity characterizing level statistics of eigenstates and is defined by Eq. (25); (ii) subsystem size $L_{\mathrm{A}}$ dependence of the entanglement entropy $S$ of an eigenstate with nonzero energy density; (iii) time evolution of the entanglement entropy of subsystem $A$ after a quench from a product state of different sites in linear timescale (left panel) and logarithmic timescale (right panel); (iv) time evolution of a physical observable $\hat{O}$ after a quench from a generic initial state.

\section{A. Level statistics}

We first focus on the eigenstate properties. It is known that energy-level spacings obey the Wigner-Dyson distribution in the ETH regime and the Poisson distribution in the MBL regime [51]. To determine the specific distribution, a dimensionless quantity $r$ has been introduced [57]

$$
r=\left\langle r_{n}\right\rangle=\left\langle\frac{\min \left(\delta_{n}, \delta_{n-1}\right)}{\max \left(\delta_{n}, \delta_{n-1}\right)}\right\rangle,
$$

where $n$ is the ascending energy level index, $\delta_{n}=E_{n+1}-E_{n}$ is the energy-level spacing between two consecutive energy levels, and the average, in our case, is taken over all energy levels, including a first average over all the eigenstates in a given symmetry sector and then a second average over different symmetry sectors. It can be shown that $r \approx 0.53$ for the Wigner-Dyson distribution and $r \approx 0.39$ for the Poisson distribution [57].

We use the exact diagonalization method with the open boundary condition to compute all eigenvalues and determine the value of $r$. As can been seen from Fig. 3, the general trend is that when $g$ becomes larger, the value of $r$ approaches 0.53 , and when $g$ is small, the value of $r$ is around 0.39. By comparing Figs. 3(a) and 3(b), one can see that when $h$ is larger, it also requires a larger $g$ to reach the ETH regime where $r \approx 0.53$. This agrees with our expectation that the (a)

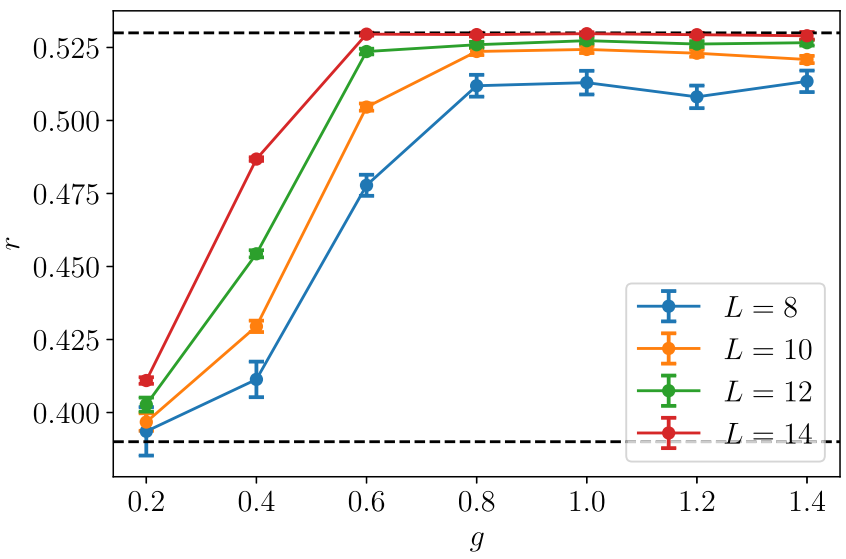

(b)

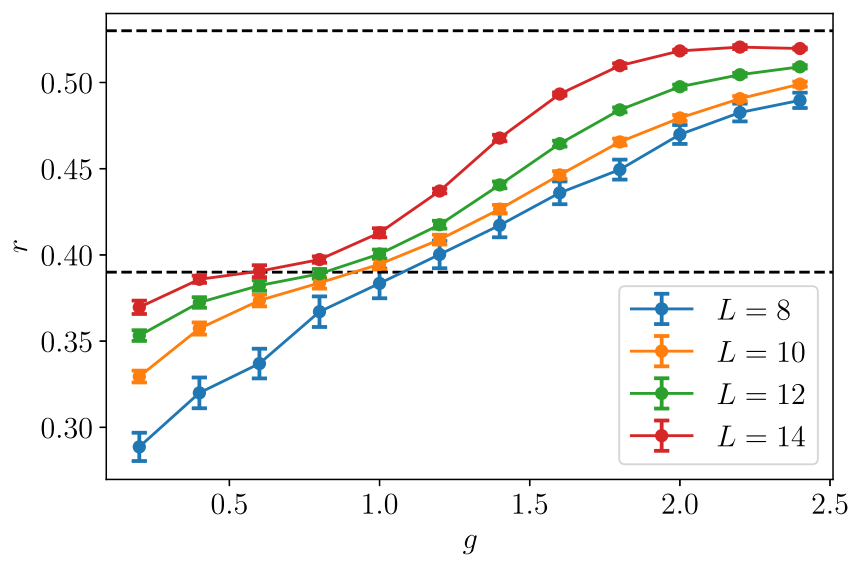

FIG. 3. The value of $r$, defined in Eq. (25), as a function of $g$ for system sizes $L=8,10,12$, and 14 at (a) $h=0.2$ and (b) $h=2.0$. The error bar stands for one standard deviation when averaging over symmetry sectors. The two dashed black lines are $r=0.39$ and 0.53 lines.

system is driven from the ETH regime to the MBL regime as the relative strength of disorder increases.

\section{B. Entanglement entropies for eigenstates}

How the von Neumann entanglement entropy $S^{v}$ of a generic eigenstate with nonzero energy density scales with the size of the subsystem $L_{A}$ is another way to differentiate the ETH regime from the MBL regime. The ETH ansatz implies the reduced density matrix of a small subsystem $A$ approaches its thermal density matrix at the temperature $T$ fixed by the energy of the eigenstate, leading to the volume law for $S^{v}$ $[55,56]$. In contrast, a MBL system fails to thermalize, and the entanglement entropy obeys the area law $[55,56]$.

The above difference is schematically shown in Fig. 2, and the behavior of $S^{v}$ is also verified in our numerical studies. As can be seen from Fig. 4(a), when $h$ is small compared with $g$, the entanglement entropy increases almost linearly with $L_{A}$ until $L_{A}$ approaches half of the system size, confirming the volume law. In contrast, as shown in Fig. 4(b), when $h$ is large compared with $g$, the entanglement entropy tends to quickly saturate as $L_{\mathrm{A}}$ increases, demonstrating the area law. 


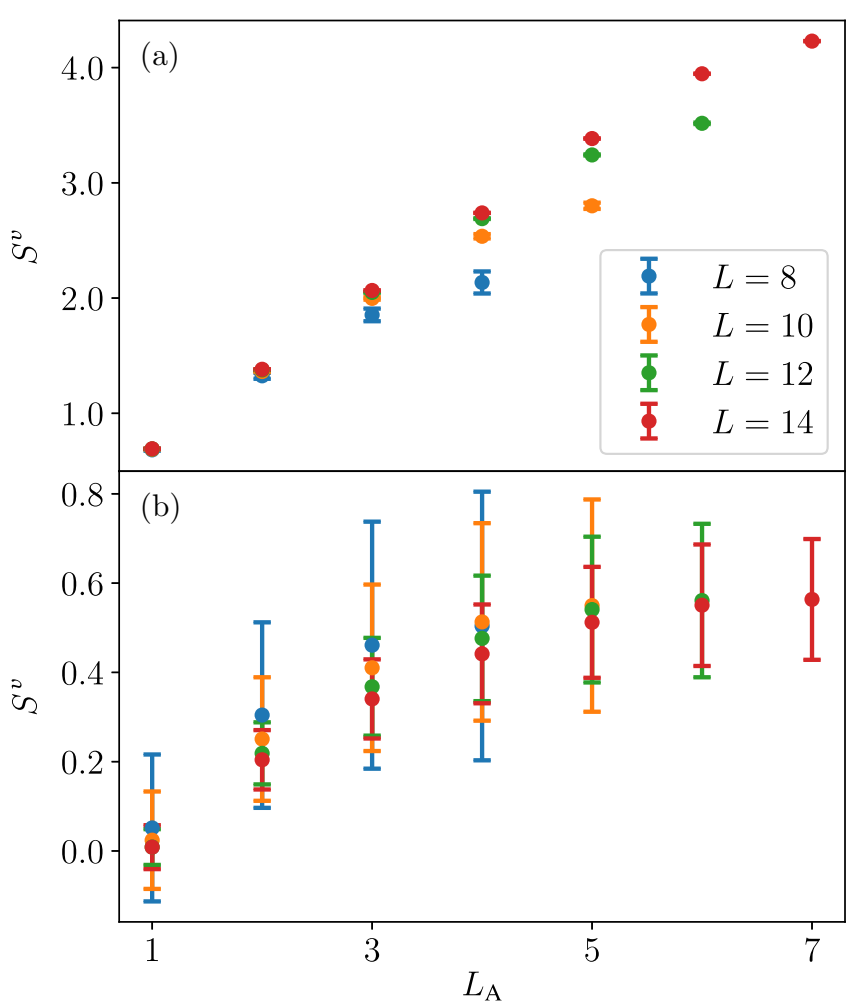

FIG. 4. The von Neumann entanglement entropy $S^{v}$ as a function of subsystem size $L_{A}$ averaged over eigenstates within a small energy window $[E, E+\Delta E]$, where $E=-1$ and $\Delta E=0.1$, at (a) $(g, h)=(1.0,0.2)$ and (b) $(g, h)=(0.2,2.0)$. Data for system sizes $L=8,10,12$, and 14 are presented, and error bars stand for one standard deviation.

\section{Entanglement entropy growth after a quench}

Aside from eigenstate properties, the differences between the ETH regime and the MBL regime also manifest themselves in quench dynamics. Starting with a product state of different sites, the entanglement entropy of a subsystem $A$ will increase from zero. In the ETH regime, the entanglement entropy increases linearly until it saturates [58]. In the MBL regime, following a short linear growth, the entanglement entropy will undergo a slow logarithmic increase in an extended time region [59-62]. This logarithmic increase is often seen as the hallmark of the MBL phase and can be explained by the phenomenological theory of MBL $[63,64]$. Yet, this linear versus logarithmic growth of entropy is also directly related to the exponential versus power-law behavior of the out-of-timeordered correlators [65-70].

Our initial product state is prepared with all $\boldsymbol{\tau}_{i}$ spins polarized along the $x$ direction and all $\sigma_{i}$ spins aligned upward or downward in a domain-wall fashion, as illustrated in the inset of Figs. 5(b) and 6(b). In terms of the original $\boldsymbol{\tau}_{i}$ spins before rotation, Eq. (20), all $\boldsymbol{\tau}_{i}$ spins are actually polarized along the $z$ direction. That is to say, all $f$ and $d$ atoms are prepared in the left wells, and all $a$ and $b$ atoms are localized either in the left ( $\sigma_{i}^{z}$ upward) or right wells ( $\sigma_{i}^{z}$ downward). The advantage of choosing such an initial state is twofold. First, the wave function is an equal-weight superposition of states from all symmetry sectors. Second, the initial state has

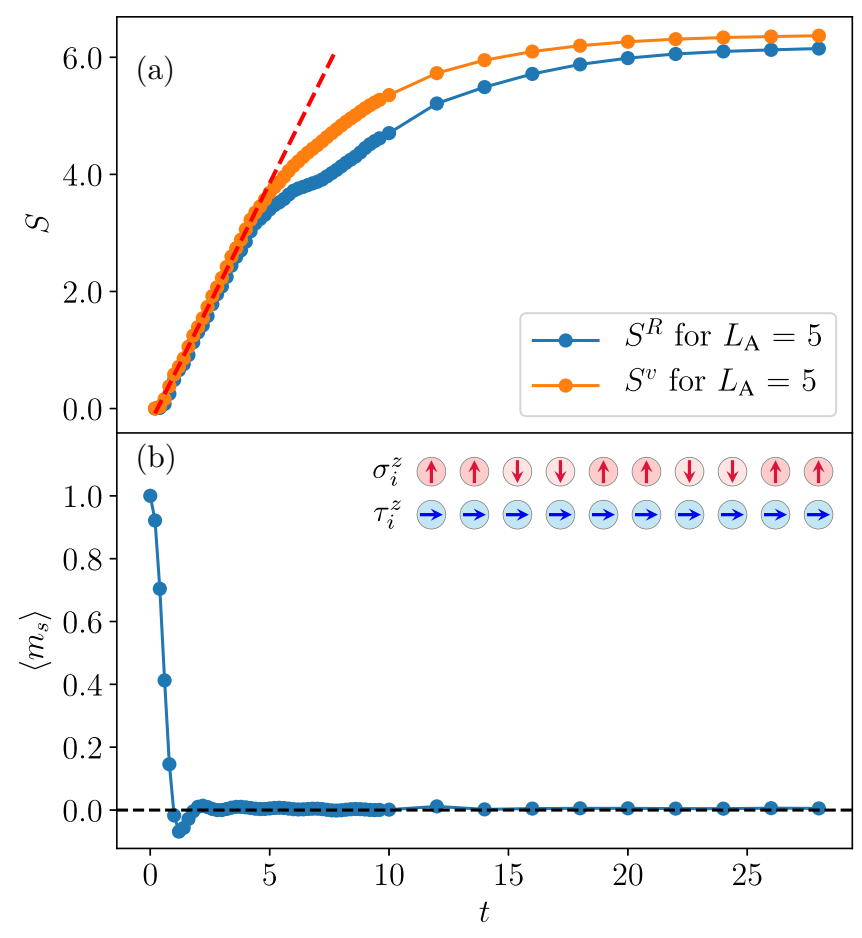

FIG. 5. Quench dynamics of (a) entanglement entropies and (b) the staggered magnetization defined in Eq. (26) from an initial product state illustrated in the inset of (b). Both the von Neumann entropy $S^{v}$ and the Rényi entropy $S^{R}$ are plotted. Here $t$ actually stands for dimensionless $4 t U$ in terms of original parameters. The system size is $L=10$ and the size of subsystem $A$ is $L_{A}=5$. The simulation is performed at $(g, h)=(1.0,0.2)$ which is shown to be inside the ETH regime.

a relative high energy which mitigates the problem of limited accessible system sizes in numerical simulations.

We numerically study the growth of both the von Neumann entanglement entropy $S^{v}$ and the second-order Rényi entropy $S^{R}$ of the subsystem $A$ that is chosen as half of the entire system. The presence of both the ETH regime and the MBL regime is demonstrated using two representative parameter sets, $(g, h)=(1.0,0.2)$ and $(g, h)=(0.2,1.0)$, as shown in Figs. 5 and 6, respectively. From Fig. 5(a), one can see that for the former case where the disorder ( $h$ term) is relatively weak, two entanglement entropies both increase linearly in time, as indicated by the red dashed line. In contrast, for the latter case where the disorder is relatively strong, a logarithmic growth of both entanglement entropies is revealed, as demonstrated by the red dashed lines in Fig. 6(a). The characteristic saturation time is also much longer than the former case.

\section{Evolution of observable after a quench}

Finally, we study the time evolution of physical observables following a quench. If a system is thermal, all local physical observables will evolve toward their thermal equilibrium values. For a MBL system, local physical observables, however, keep part of the memory of the initial state and do not necessarily approach the thermal equilibrium values. 


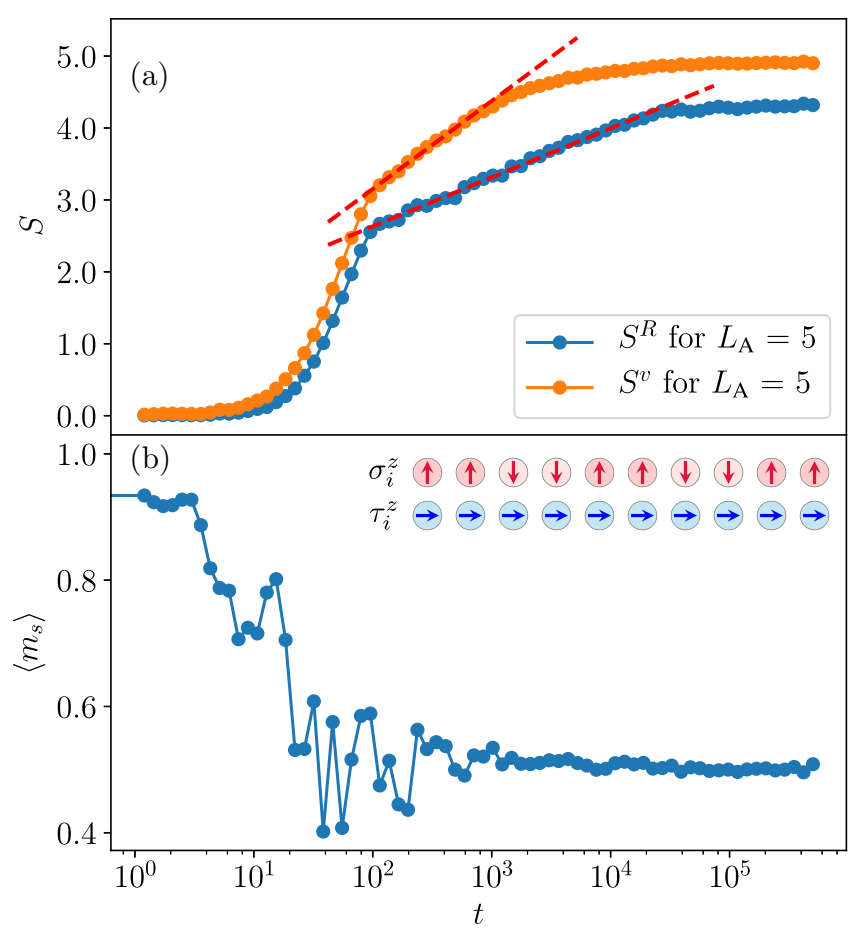

FIG. 6. Quench dynamics of (a) entanglement entropies and (b) the staggered magnetization defined in Eq. (26) from an initial product state illustrated in the inset of (b). Both the von Neumann entropy $S^{v}$ and the Rényi entropy $S^{R}$ are plotted in logarithmic timescale. Here, $t$ actually stands for dimensionless $4 t U$ in terms of original parameters. The system size is $L=10$ and the size of subsystem $A$ is $L_{A}=5$. The simulation is performed at $(g, h)=(0.2,1.0)$ which is shown to be inside the MBL regime.

This criterion has been applied in identifying MBL phases in cold-atom experiments [71].
Here, we consider a specific observable, the staggered magnetization, whose definition depends on the initial state

$$
\hat{m}_{s}=\frac{2}{L} \sum_{i} \xi_{i} \hat{\sigma}_{i}^{z},
$$

where $\xi_{i}=1$ if initially $\sigma_{i}^{z}=\frac{1}{2}$ and $\xi_{i}=-1$ if initially $\sigma_{i}^{z}=$ $-\frac{1}{2}$. The advantage of defining such an observable is also twofold. First, the initial expectation value of our observable is always $m_{s}=1$. Second, it is easy to show that the thermal average of $\hat{m}_{s}$ is always zero because of the $\hat{\sigma}_{i}^{z} \rightarrow-\hat{\sigma}_{i}^{z}, q_{i} \rightarrow$ $-q_{i}$ symmetry of the Hamiltonian, under which $m_{s}$ changes its sign. By comparing Figs. 5(b) and 6(b), it is clear that the system thermalizes in the former case but fails to thermalize in the latter case.

\section{OUTLOOK}

In summary, we have established that many-body localization can occur in a disorder-free system with local gauge symmetries. In our model, we explicitly show that gauge charges of different symmetry sectors can play the role of disorder potentials. All together with recent efforts [43-46], we would like to conclude this work by posting the following question. Is the many-body localization a generic feature of all dynamical gauge theories with local gauge symmetries? If yes, can this development can also shed light on studying lattice gauge theories in high-energy physics?

\section{ACKNOWLEDGMENTS}

This work is supported by Beijing Outstanding Young Scientist Program, MOST under Grant No. 2016YFA0301600 and NSFC Grant No. 11734010.
[1] V. Galitski, G. Juzeliūnas, and I. B. Spielman, Phys. Today 72(1), 38 (2019).

[2] N. Goldman, G. Juzeliūnas, P. Öhberg, and I. B. Spielman, Rep. Prog. Phys. 77, 126401 (2014).

[3] J. Dalibard, F. Gerbier, G. Juzeliūnas, and P. Öhberg, Rev. Mod. Phys. 83, 1523 (2011).

[4] Y. J. Lin, R. L. Compton, A. R. Perry, W. D. Phillips, J. V. Porto, and I. B. Spielman, Phys. Rev. Lett. 102, 130401 (2009).

[5] J. Struck, C. Ölschläger, M. Weinberg, P. Hauke, J. Simonet, A. Eckardt, M. Lewenstein, K. Sengstock, and P. Windpassinger, Phys. Rev. Lett. 108, 225304 (2012).

[6] Y. J. Lin, R. L. Compton, K. Jiménez-García, J. V. Porto, and I. B. Spielman, Nature (London) 462, 628 (2009).

[7] Y. J. Lin, R. L. Compton, K. Jiménez-García, W. D. Phillips, J. V. Porto, and I. B. Spielman, Nat. Phys. 7, 531 (2011).

[8] Y. J. Lin, K. Jiménez-García, and I. B. Spielman, Nature (London) 471, 83 (2011).

[9] P. Wang, Z.-Q. Yu, Z. Fu, J. Miao, L. Huang, S. Chai, H. Zhai, and J. Zhang, Phys. Rev. Lett. 109, 095301 (2012).
[10] L. W. Cheuk, A. T. Sommer, Z. Hadzibabic, T. Yefsah, W. S. Bakr, and M. W. Zwierlein, Phys. Rev. Lett. 109, 095302 (2012).

[11] L. J. LeBlanc, K. Jiménez-García, R. A. Williams, M. C. Beeler, A. R. Perry, W. D. Phillips, and I. B. Spielman, Proc. Natl. Acad. Sci. U. S. A. 109, 10811 (2012).

[12] V. Galitski and I. B. Spielman, Nature (London) 494, 49 (2013).

[13] H. Zhai, Int. J. Mod. Phys. B 26, 1230001 (2012)

[14] H. Zhai, Rep. Prog. Phys. 78, 026001 (2015).

[15] C. Wang, C. Gao, C.-M. Jian, and H. Zhai, Phys. Rev. Lett. 105, 160403 (2010).

[16] T.-L. Ho and S. Zhang, Phys. Rev. Lett. 107, 150403 (2011).

[17] Y. Li, G. I. Martone, L. P. Pitaevskii, and S. Stringari, Phys. Rev. Lett. 110, 235302 (2013).

[18] S.-C. Ji, J.-Y. Zhang, L. Zhang, Z.-D. Du, W. Zheng, Y.-J. Deng, H. Zhai, S. Chen, and J.-W. Pan, Nat. Phys. 10, 314 (2014).

[19] J.-R. Li, J. Lee, W. Huang, S. Burchesky, B. Shteynas, F.Ç. Top, A. O. Jamison, and W. Ketterle, Nature (London) 543, 91 (2017). 
[20] N. R. Cooper, J. Dalibard, and I. B. Spielman, Rev. Mod. Phys. 91, 015005 (2019).

[21] F. Görg, K. Sandholzer, J. Minguzzi, R. Desbuquois, M. Messer, and T. Esslinger, Nat. Phys. 15, 1161 (2019).

[22] L. W. Clark, B. M. Anderson, L. Feng, A. Gaj, K. Levin, and C. Chin, Phys. Rev. Lett. 121, 030402 (2018).

[23] C. Schweizer, F. Grusdt, M. Berngruber, L. Barbiero, E. Demler, N. Goldman, I. Bloch, and M. Aidelsburger, Nat. Phys. 15, 1168 (2019).

[24] L. Barbiero, C. Schweizer, M. Aidelsburger, E. Demler, N. Goldman, and F. Grusdt, Sci. Adv. 5, eaav7444 (2019).

[25] F. M. Surace, P. P. Mazza, G. Giudici, A. Lerose, A. Gambassi, and M. Dalmonte, Phys. Rev. X 10, 021041 (2020).

[26] A. Mil, T. V. Zache, A. Hegde, A. Xia, R. P. Bhatt, M. K. Oberthaler, P. Hauke, J. Berges, and F. Jendrzejewski, Science 367, 1128 (2020).

[27] B. Yang, H. Sun, R. Ott, H.-Y. Wang, T. V. Zache, J. C. Halimeh, Z.-S. Yuan, P. Hauke, and J.-W. Pan, arXiv:2003.08945.

[28] E. Zohar, J. I. Cirac, and B. Reznik, Phys. Rev. Lett. 109, 125302 (2012).

[29] L. Tagliacozzo, A. Celi, A. Zamora, and M. Lewenstein, Ann. Phys. 330, 160 (2013).

[30] D. Banerjee, M. Dalmonte, M. Müller, E. Rico, P. Stebler, U.-J. Wiese, and P. Zoller, Phys. Rev. Lett. 109, 175302 (2012).

[31] E. Zohar, J. I. Cirac, and B. Reznik, Phys. Rev. Lett. 110, 125304 (2013).

[32] L. Tagliacozzo, A. Celi, P. Orland, M. Mitchell, and M. Lewenstein, Nat. Commun. 4, 2615 (2013).

[33] D. Banerjee, M. Bögli, M. Dalmonte, E. Rico, P. Stebler, U.-J. Wiese, and P. Zoller, Phys. Rev. Lett. 110, 125303 (2013).

[34] U.-J. Wiese, Ann. Phys. 525, 777 (2013).

[35] M. Dalmonte and S. Montangero, Contemp. Phys. 57, 388 (2016).

[36] E. Zohar, J. I. Cirac, and B. Reznik, Rep. Prog. Phys. 79, 014401 (2016).

[37] E. A. Martinez, C. A. Muschik, P. Schindler, D. Nigg, A. Erhard, M. Heyl, P. Hauke, M. Dalmonte, T. Monz, P. Zoller, and R. Blatt, Nature (London) 534, 516 (2016).

[38] C. Muschik, M. Heyl, E. Martinez, T. Monz, P. Schindler, B. Vogell, M. Dalmonte, P. Hauke, R. Blatt, and P. Zoller, New J. Phys. 19, 103020 (2017).

[39] V. Kasper, F. Hebenstreit, F. Jendrzejewski, M. K. Oberthaler, and J. Berges, New J. Phys. 19, 023030 (2017).

[40] M. C. Bañuls, R. Blatt, J. Catani, A. Celi, J. I. Cirac, M. Dalmonte, L. Fallani, K. Jansen, M. Lewenstein, S. Montangero, C. A. Muschik, B. Reznik, E. Rico, L. Tagliacozzo, K. Van Acoleyen, F. Verstraete, U.-J. Wiese, M. Wingate, J. Zakrzewski, and P. Zoller, Eur. Phys. J. D 74, 165 (2020).

[41] A. Russomanno, S. Notarnicola, F. M. Surace, R. Fazio, M. Dalmonte, and M. Heyl, Phys. Rev. Res. 2, 012003 (2020).

[42] U. Borla, R. Verresen, F. Grusdt, and S. Moroz, Phys. Rev. Lett. 124, 120503 (2020).
[43] A. Smith, J. Knolle, D. L. Kovrizhin, and R. Moessner, Phys. Rev. Lett. 118, 266601 (2017).

[44] A. Smith, J. Knolle, R. Moessner, and D. L. Kovrizhin, Phys. Rev. Lett. 119, 176601 (2017).

[45] A. Smith, J. Knolle, R. Moessner, and D. L. Kovrizhin, Phys. Rev. B 97, 245137 (2018).

[46] M. Brenes, M. Dalmonte, M. Heyl, and A. Scardicchio, Phys. Rev. Lett. 120, 030601 (2018).

[47] E. Fradkin, Field Theories of Condensed Matter Physics (Cambridge University Press, Cambridge, 2013).

[48] J. M. Deutsch, Phys. Rev. A 43, 2046 (1991).

[49] M. Srednicki, J. Phys. A: Math. Gen. 29, L75 (1996).

[50] M. Rigol, V. Dunjko, and M. Olshanii, Nature (London) 452, 854 (2008).

[51] L. D'Alessio, Y. Kafri, A. Polkovnikov, and M. Rigol, Adv. Phys. 65, 239 (2016).

[52] I. V. Gornyi, A. D. Mirlin, and D. G. Polyakov, Phys. Rev. Lett. 95, 206603 (2005).

[53] D. M. Basko, I. L. Aleiner, and B. L. Altshuler, Ann. Phys. 321, 1126 (2006).

[54] D. J. Luitz, N. Laflorencie, and F. Alet, Phys. Rev. B 91, 081103(R) (2015).

[55] R. Nandkishore and D. A. Huse, Annu. Rev. Condens. Matter Phys. 6, 15 (2015).

[56] D. A. Abanin, E. Altman, I. Bloch, and M. Serbyn, Rev. Mod. Phys. 91, 021001 (2019).

[57] V. Oganesyan and D. A. Huse, Phys. Rev. B 75, 155111 (2007).

[58] H. Kim and D. A. Huse, Phys. Rev. Lett. 111, 127205 (2013).

[59] M. Žnidarič, T. Prosen, and P. Prelovšek, Phys. Rev. B 77, 064426 (2008).

[60] J. H. Bardarson, F. Pollmann, and J. E. Moore, Phys. Rev. Lett. 109, 017202 (2012).

[61] M. Serbyn, Z. Papić, and D. A. Abanin, Phys. Rev. Lett. 110, 260601 (2013).

[62] R. Vosk and E. Altman, Phys. Rev. Lett. 110, 067204 (2013).

[63] D. A. Huse, R. Nandkishore, and V. Oganesyan, Phys. Rev. B 90, 174202 (2014).

[64] M. Serbyn, Z. Papić, and D. A. Abanin, Phys. Rev. Lett. 111, 127201 (2013).

[65] R. Fan, P. Zhang, H. Shen, and H. Zhai, Sci. Bull. 62, 707 (2017).

[66] B. Swingle, G. Bentsen, M. Schleier-Smith, and P. Hayden, Phys. Rev. A 94, 040302(R) (2016).

[67] Y. Chen, arXiv:1608.02765.

[68] X. Chen, T. Zhou, D. A. Huse, and E. Fradkin, Ann. Phys. (Berlin) 529, 1600332 (2017).

[69] Y. Huang, Y. L. Zhang, and X. Chen, Ann. Phys. (Berlin) 529, 1600318 (2017).

[70] R.-Q. He and Z.-Y. Lu, Phys. Rev. B 95, 054201 (2017).

[71] M. Schreiber, S. S. Hodgman, P. Bordia, H. P. Lüschen, M. H. Fischer, R. Vosk, E. Altman, U. Schneider, and I. Bloch, Science 349, 842 (2015). 